\title{
Kritische Betrachtung rund um das Thema Organtransplantation
}

\section{Werner Niederer}

Dr. med., emeritierter Leiter der Ophthalmochirurgie am Spitalzentrum Biel

\author{
Die Nationale Ethik-Kommission hat die Forderung der JCI-Initiative als ethisch \\ fragwürdig taxiert. Eine im November durchgeführte gfs-Umfrage zeigt, dass in \\ Bezug auf das Verständnis des Hirntodes ein grosses Informationsdefizit in der Be- \\ völkerung besteht. Deshalb sollte der Entscheid, ob und unter welchen Bedingun- \\ gen jemand seine Organe zur Transplantation freigibt, faktenbasiert und unter Ein- \\ bezug aller Tatsachen erfolgen und nicht aufgrund professioneller PR-Massnahmen.
}

Ich möchte (als Ergänzung zu meinem Artikel «Kritik zu den Richtlinien zur Feststellung des Todes mit Bezug auf Organtransplantationen der SAMW» (Schweizerische Ärztezeitung 2002 Nr. 49) auf weitere Argumente hinweisen, die gegen die Transplantation von Organen eines «Hirntoten» sprechen. Vorausschicken möchte ich, dass ich betreffend Organentnahme von Toten (z.B. Cornea) und von Lebenden (z.B. Knochenmark oder eine von zwei Nieren) keinerlei ethischen Probleme sehe.

\section{Kritik an der Werbung für Organspende}

In den Erklärungen zur JCI-Volksinitiative liest man oft: «Wegen Organmangel stirbt in der Schweiz jeden zweiten Tag ein Mensch» (die Angaben variieren ein bisschen). Solche Information grenzt an Irreführung. Die Menschen, die vergeblich auf eine Transplantation gewartet haben, sterben wegen ihrer Krankheit. Viel-

\section{Nur wenn eine Operation besonders gute Resultate zeitigt, wird der Patient zum Vorzeigefall.}

leicht darf man in gewissen Fällen annehmen, dass der eine oder andere Patient später gestorben wäre, wenn ein Organ zur Verfügung gestanden wäre. Wie aber die Frequenz «alle zwei Tage» ermittelt wurde, bleibt unklar. Die Patienten, die so schwer krank sind, dass sie in den nächsten 2-4 Tagen sterben, stehen ja vermutlich nicht mehr ganz oben auf der Warteliste der potentiellen Organempfänger. Die Lebenserwartung der operablen Kranken bei medikamentöser und maschineller
Therapie wird verschwiegen. Ebenfalls verschwiegen wird, dass z.B. nach einer Herztransplantation jeder vierte Patient innerhalb der ersten drei Jahre stirbt (ca. 25\%, vgl. Database UniversitätsSpital Zürich 2014). Betreuungsaufwand, Nachbehandlung, Leistungsfähigkeit und Wohlbefinden der Operierten werden selten dargelegt. Nur wenn eine Operation besonders gute Resultate zeitigt, wird der Patient zum Vorzeigefall. So begegnet man z.B. der Patientin Renata Isenschmid nicht nur in srf-Sendungen, sondern auch in der NZZ, in den News von press24.net, auffacebook, im Beobachter und natürlich auch im Magazin von Swisstransplant. Im Beobachter sagt die 57-jährige Patientin sieben Jahre nach ihrer Herzimplantation: «Ich habe es gut. Eigentlich kann ich machen, was ich will. In meinen superguten Phasen könnte ich Bäume ausreissen, so viel Power habe ich. Dann gibt es Zeiten, da habe ich null Energie - und weiss nicht, warum. Mein Partner, Familie und Freunde waren mir immer eine riesige Stütze, auch heute bin ich auf sie angewiesen. Es muss sehr traurig sein, allein dazustehen [...] Wer glaubt, nach der Operation sei alles gut, irrt - leider. Es ist nie zu Ende. Ständig Medikamente, einmal im Monat zur Hausärztin, alle sechs Monate nach Bern zur Nachkontrolle. Die Angst, dass die Werte einmal schlecht sind oder sie etwas Negatives entdecken, sitzt mir immer ein wenig im Nacken [...] Dass ein transplantiertes Herz nur 10, 15 Jahre hält, sagte man mir zwar schon im Spital, aber ich vergass es wieder.» Eine Mitteilung von Renata Isenschmid ist besonders bedenkenswert: Sie war präoperativ an einer künstlichen Blutpumpe angeschlossen. Tatsächlich gibt es auch schon Pumpen, die man implantieren kann. Es scheint mir einleuchtend, 
dass Organmangel stärker als Organüberfluss die entsprechenden Unternehmen motiviert, Pumpen zu entwickeln, die ebenso gut funktionieren wie die Herzen von Organspendern.

In den Schweizer Medien wird gejammert, das Organangebot habe abgenommen, weil es infolge besserer Verkehrsregulierung weniger Hirntote gebe. Das klingt, wenn auch sicher ungewollt, fast etwas zynisch, da es implizit besagt, tödliche Unfälle hätten auch etwas Gutes an sich. Auch die Werbung von Swisstransplant bekommt einen sarkastischen Touch, wenn das Jesuswort «Geben ist seliger als Nehmen» (Apg. 29,15) im Zusammenhang mit der Organtransplantation zitiert wird. Ähnlich unpassend wäre es, Jesus zu zitieren, der gemäss Joh 15,13 gesagt hat, es gebe keine grössere Liebe als die, dass jemand sein Leben hingibt für seine Freunde. Aufruf zum Suizid aus Nächstenliebe, um Organe zu spenden? Sicher eine absurde Idee, weil Selbstmord für alle Angehörigen und Freunde wie ein liebloser, verächtlicher Schlag ins Gesicht empfunden wird.

\section{Kritik an der Verwendung der Organe von anonymen «Hirntoten"}

Meine Argumente gegen Organtransplantationen sind weder religiöser noch esoterischer Natur. Vielmehr

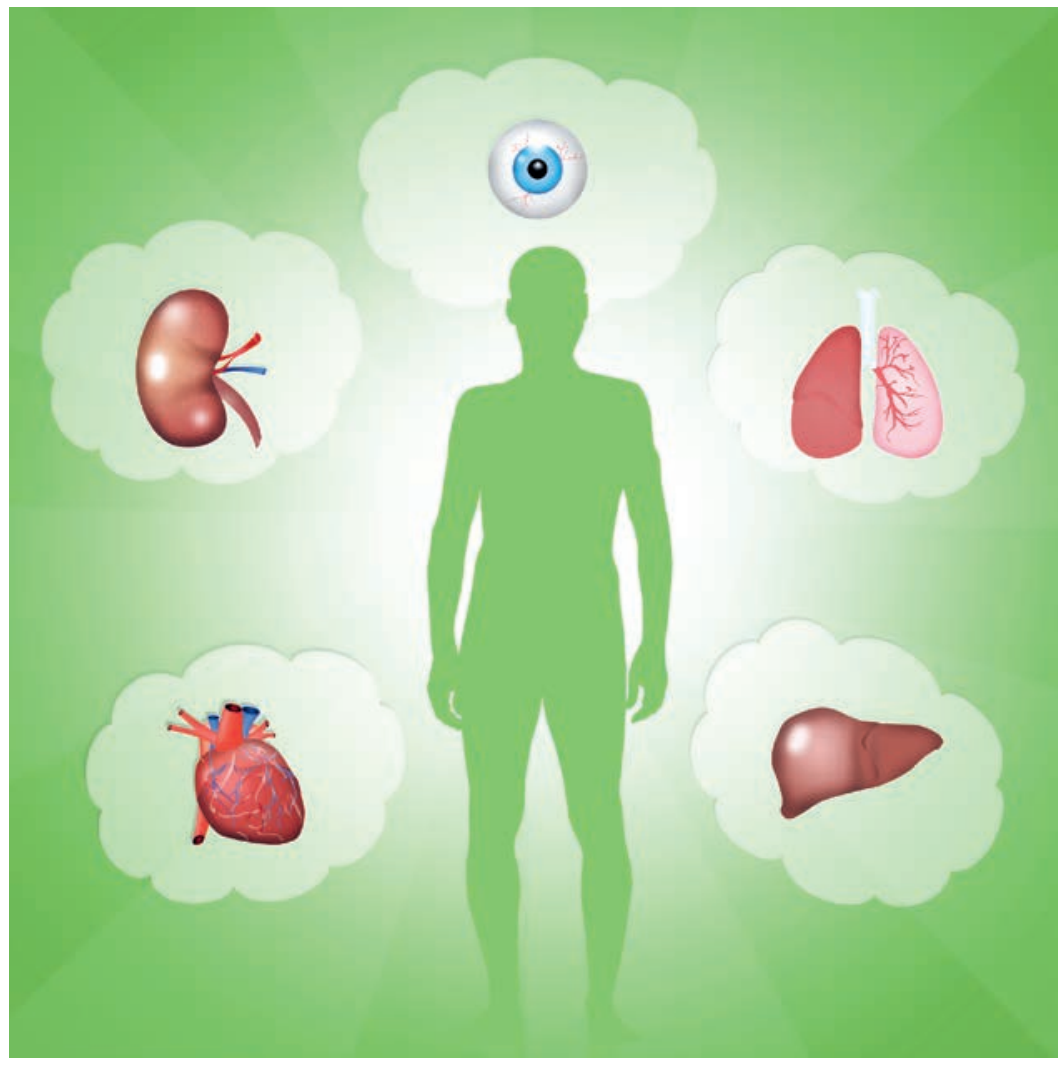

Die Lebensdauer eines transplantierten Organs ist beschränkt.

(C) Adrenalinapura | Dreamstime.com) denke ich an die wissenschaftlich belegbare Tatsache, dass die Persönlichkeit eines Menschen keineswegs alleine vom Hirn bestimmt wird. Es ist bekannt, dass zum Beispiel Hormone das Denken stark beeinflussen. Die psychische Veränderung durch zu viel oder zu wenig Nebennierenrindenhormone sind wohlbekannt. Ebenso die Einflüsse der Sexualhormone auf die Denkund Verhaltensweisen. Die Steuerung über die Hypophyse bzw. über die Releasing-Faktoren der Epiphyse ist ein komplexes Feedback-System, das den ganzen Menschen auch psychisch beeinflusst. Es ist bekannt, dass nicht nur Angst eine Adrenalinausschüttung und damit eine Tachykardie auslöst, sondern auch umgekehrt eine organisch bedingte Tachykardie zu Angstzuständen führt. Erst solche und viele andere bekannte und sicher auch noch unbekannte Wechselwirkungen zwischen Organen, Hirn und Psyche ergeben eine Persönlichkeit. Bei Patienten mit «Locked-inSyndrom" sind nur noch die neuronalen Verbindungen des Hirns mit dem Kopf vorhanden; und dies hat eine verblüffende Gleichmut zur Folge; depressive $\mathrm{Zu}$ stände, die man eigentlich erwartet, bleiben aus. Das kann nur damit erklärt werden, dass die psychische Verfassung mitbestimmt wird von neuralen Rückmeldungen des Körpers zum Gehirn. Von einem Menschen mit einem Herzen, das auf Adrenalin überdurchschnittlich schnell zu Tachykardie neigt, wird man sagen, er sei eben eine ängstliche Persönlichkeit. Im Gegensatz dazu wird ein subklinisch Hypothyreoter den Ruf haben, eine gelassene Person zu sein, usw.

\section{Gestalttheoretische Kritik}

Man darf annehmen, dass wir längst nicht alle Wechselwirkungen zwischen Leib und Psyche kennen. Für jene, welche die Verwendung des Begriffs «Psyche» schon als "esoterisch" diffamieren, weil sie nicht an eine Psyche im Sinne einer selbständig existierenden Entität glauben, mag man statt von der Psyche auch vom psychischen Verhalten sprechen. (Nebenbei: Die Einheit von Leib und Geist entspricht auch dem christlichen Glauben; andernfalls würde dieser nicht die Auferstehung lehren.) Kurz: Das Zusammenwirken aller Organe (nicht das Hirn alleine) ergibt eine einmalige Kooperation, die zusammen mit der Biographie eine Person prägt. «Das Ganze ist mehr als die Summe seiner Teile», lehrt die Gestalttheorie (Max Wertheimer), die leider zu wenig Eingang in medizinethische Diskussionen gefunden hat. Die Verfechter von Organtransplantationen verschweigen, dass nicht alleine das Hirn eine Person ausmacht, sondern das Zusammenspiel aller Organe inklusive der Haut, die sich streicheln lässt, 
und der Ohren, die beim Hören schöner Musik das Herz erwärmen. Es gibt zahlreiche Redewendungen wie «das war ein herzloses Verhalten", "er ist ein Choleriker», "etwas ist ihm über die Leber gekrochen", «diese Sorge liegt ihm auf dem Magen» usw. Sind solche Redewendungen etwa bloss entstanden, weil man von den Funktionen der inneren Organe keine Ahnung hatte? Vor einem Jahr wurde in den Medien ein Fall diskutiert, dass ein Gefängnisinsasse Beihilfe von Exit gefordert habe, um sich umzubringen. Wäre nun ein Wartelistenpatient einverstanden, das Herz des Gefängnisinsassen zu bekommen, wenn er über den Spender aufgeklärt würde? Und was wäre die Haltung der Chirurgen? Eher ja bei einem Mörder oder bei einem Bankräuber?

\section{Kritik an der heuristischen Perzeption der Vergänglichkeit in der Medizin}

Die Transplantationschirurgie lebt von der Angst der Menschen vor dem Sterben. Ich spreche jetzt nicht von der reflexartigen Angst in Todesgefahr, wie sie auch alle Tiere haben; diese Angst besteht ja nur momentan als Schutzreflex und ist eine evolutive Errungenschaft der Lebewesen, die in Todesgefahr Höchstleistungen vollbringen müssen, um zu überleben und sich weiter fortzupflanzen. Hier aber geht es mir um die Angst vor dem Tod beim Bedenken der Unausweichlichkeit desselben. Dass jeder Mensch mehr oder weniger häufig die Vergänglichkeit bedenkt, gibt ihm eine ganz besondere Stellung unter den Lebewesen. Solches Sinnieren muss nicht hochtrabendes Philosophieren sein, sollte sich aber auch nicht mit primitiven Feststellungen begnügen wie «das Leben ist so etwas Tolles, also koste es aus, koste es, was es wolle!» oder "das Leben ist eine Hühnerleiter: kurz und beschissen!».

Das Nachdenken über den Tod ist das Nachdenken über den Sinn des Lebens oder noch allgemeiner: über den Sinn des Seienden. Wenn wir zur Überzeugung gelan-

Dass jeder Mensch mehr oder weniger häufig die Vergänglichkeit bedenkt, gibt ihm eine ganz besondere Stellung unter den Lebewesen.

gen, alles Sein habe einen verborgenen Sinn (also auch das persönliche Dasein), dann muss es einen unvergänglichen Sinn (einen Ewigkeitswert, eine Bedeutung im Zeitlosen) haben. Andernfalls gäbe es das Sein gar nicht, sondern nur die imaginäre Grenze zwischen Vergangenheit und Zukunft. Also muss auch das individuelle Leben im Zeitlosen eine Bedeutung, eine Existenz, eine hinterlassene Spur haben. Das sind ontologische Einsichten die man nicht über die Hühnerleiter gewinnt.

Und nun das Wunder: Ziemlich einmütig sagen alle Menschen, die an den Sinn (und damit an die Unvergänglichkeit) des Seienden glauben: Das Ewige eines menschlichen Individuums kann nur seine gelebte Liebe sein. Aber ist das nun ein Argument für oder gegen die Organentnahme bei «Hirntoten»? Die Antwort muss jeder Mensch selbst finden. Er findet sie nur, wenn er über Tod und Liebe nachdenkt. Mit zur richtigen Antwort gehört vermutlich, dass man sie nicht für die einzig richtige hält.
Dr. med. Werner Niederer Sternenmatte 13 CH-3293 Dotzigen niederer.werner[at]gmx.ch

\section{Das Wichtigste in Kürze}

- Die JCl-Initiative zur Förderung der Organspende wurde durch die Nationale Ethik-Kommission als ethisch fragwürdig taxiert, da sie die Persönlichkeitsrechte von sich passiv verhaltenden Bürgern verletze.

- Die Transplantationschirurgie lebt von der Angst der Menschen vor dem Sterben. Information der Öffentlichkeit erfolgt nicht transparent und ist oft irreführend.

- Diverse psychoorganische Syndrome weisen darauf hin, dass nicht das Hirn allein, sondern das Zusammenspiel aller Organe einer Person deren Persönlichkeit prägen. Diese Erkenntnis sollte im Sinne von Wertheimers Gestalttheorie bedacht werden, bevor man Organe transplantiert.

\section{L'essentiel en bref}

- La Commission nationale d'éthique a jugé que l'initiative de la $\mathrm{JCl}$ pour favoriser le don d'organes lèse les droits de la personnalité des citoyens passifs et l'a donc estimée douteuse sur le plan éthique.

- La chirurgie des greffes vit de la crainte de la mort. L'information du public manque de transparence et est souvent trompeuse.

- Divers syndromes psycho-organiques montrent que la personnalité n'est pas déterminée par le cerveau seul, mais par l'interaction de tous les organes d'une personne. II serait bon de tenir compte de ce constat dans l'esprit du gestaltisme de Wertheimer avant de transplanter des organes. 